\title{
The feasibility and utility of gratitude intervention for suicidal internet users
}

\author{
Hajime Sueki ${ }^{*} \&$ Kenta Ishikawa ${ }^{2}$
}

\begin{abstract}
Background: Gratitude has received attention as a protective factor that contributes to suicide prevention, and several studies have shown that gratitude intervention in hospitalized patients has a suicide prevention effect. Aim: We conducted a gratitude intervention for suicidal internet users, and examined the feasibility and effectiveness of the intervention. Methods: This is a single-arm study of gratitude intervention ("counting blessings") among Japanese internet users with high suicidal ideation $(N=225)$. The experiment consisted of a baseline period of approximately 4 weeks and an intervention period of approximately 4 weeks. The main factor measured was suicidal ideation.

Results: The dropout rate was $16.5 \%$ during the baseline period and $24.5 \%$ during the intervention period. Commitment to gratitude intervention was significantly associated with reduced suicidal ideation, after controlling for mean suicidal ideation in the baseline period and other demographic variables. Limitations: This study did not include a control group and a follow-up period.

Conclusions: Gratitude intervention has the potential to develop into a suicide prevention tool that can be used by suicidal internet users who do not necessarily have access to psychiatric care.
\end{abstract}

\section{Keywords:}

suicide prevention, suicidality, suicide risk, positive psychology, counting blessings

1 Faculty of Human Sciences, Wako University, 5-1-1 Kanaigaoka, Machida, Tokyo, 195-8585, Japan 2 Institute of Humanities, Senshu University, 2-1-1, Higashimita, Tama-Ku, Kawasaki, Kanagawa, 214-8580, Japan

*Corresponding author: Hajime Sueki (h_sueki@wako.ac.jp) 
One of the most integral suicide prevention strategies is strengthening protective factors against suicide. Although there is limited research on protective factors against suicide, the research focus on the relationship between gratitude and suicide prevention has increased in recent years (Kleiman et al., 2013; Krysinska et al., 2015). Gratitude is a positive interpersonal emotion that arises from the perception that one benefits from the positive intentions of others. This perception may serve as a suicide-preventive function by improving relationships with others (Ducasse et al., 2019).

To the best of our knowledge, only two studies exist on gratitude intervention for suicide prevention (Ducasse et al., 2019; Huffman et al., 2014). Huffman et al. (2014) conducted a singlearm study of positive psychological interventions including gratitude letters and counting blessings in adults hospitalized for current suicidal ideation or suicide attempts. According to their analysis, positive psychology exercises conducted for inpatients with suicidal ideation were feasible and associated with short-term reduction in hopelessness and increase in optimism. Ducasse et al. (2019) conducted a randomized controlled trial based on a 7-day program: a gratitude diary versus food diary for adults hospitalized for current suicidal ideation or a suicide attempt. The results showed that the current psychological pain reduced significantly $(d=0.29)$. In addition, suicidal ideation was reduced slightly, but the effect size $(d=0.19-0.22)$ was smaller than that of current psychological pain. From the above intervention studies, remembering and writing down events to be grateful for may function as suicide prevention.

The limitations of previous studies using gratitude intervention is that all of them were conducted on hospitalized patients who were at high risk of suicide. Since suicidal people exist outside of hospitals as well, examining the feasibility of conducting the program in a wider range of settings would contribute to suicide prevention. Therefore, in this study, we conducted a gratitude intervention on the internet for suicidal internet users and examined the feasibility and effectiveness of the program.

\section{Methods}

\section{Study Design \& Procedure}

This is a non-randomized, open-label, single-arm study of "counting blessings" (Emmons \& McCullough, 2003) among Japanese internet users with high suicidal ideation. This study was conducted with members of internet survey panels through a major Japanese internet survey company. Figure 1 shows the research design and sampling process. The screening survey was based on a target population aged 20 years or older distributed according to the demographics of the latest census data in Japan (Japanese Ministry of Internal Affairs and Communications, 2016). The stratified variables were age, sex, and geographic region of residence. 
We then included 395 participants, who scored 6 or higher on the Suicidal Ideation Scale (Sueki, 2019) in the screening survey. The experiment consisted of a baseline period of approximately 4 weeks and an intervention period of about 4 weeks. During the baseline and intervention periods, measurements (a total of eight) were recorded weekly. The intervention with the participants was conducted using the same procedures as in previous research on "counting blessings" (Emmons \& McCullough, 2003), a well-known gratitude intervention. During the intervention period, participants were asked to reflect on the events of the past week and to write down five things for which they were grateful. The responses were open-ended, and all five blank spaces had to be filled in in order to complete the questionnaire.

It was not possible to obtain a priori institutional review board approval, since the institution to which the first author belongs has no such board. However, the study was conducted in accordance with the Helsinki Declaration (revised 1989). Prior to participation, participants were advised that answering some of the survey questions may lead to a change in sentiment and that they were allowed to quit at any point in the course of the survey. Informed consent from the participants was obtained online. Additionally, participants were provided with a website with links containing professional support resources while completing the questionnaire.

\section{Measurements}

All participants completed an online, self-administered questionnaire covering suicidal ideation and demographic details (sex, age, marital status, family structure, employment, educational background, annual household income, and present psychiatric hospital visit).

Suicidal ideation was measured using the short form of the Suicidal Ideation Scale (Sueki, 2019). This scale is a shortened version of the Japanese self-administered Suicidal Ideation Scale, which is based on the Scale for Suicide Ideation designed by Beck et al. (1979). This 6-item instrument (see Appendix 1) was designed to quantify suicidal ideation. Responses are rated on a 3point scale from 0 to 2; higher scores indicate higher suicidal ideation levels (total score range: 0 12). The scale showed internal consistency (Cronbach's $\alpha=.89$ ), test-retest reliability (4-week interval: Spearman's $\rho=.84$ ), and validity in a survey with 2,486 internet users (Sueki, 2019).

\section{Statistical Analyses}

We considered participants to be properly engaged in the "counting blessings" task if they wrote an average of 15 or more Japanese characters in each free text field. We set 15 characters as the standard because this is the minimum number of characters needed to express gratitude to others in short sentences in Japanese. For example, the Japanese phrase "danna ga keki wo katte kite kureta' (My husband bought me a cake) has 15 characters. The participants who met the above criteria were defined as the commitment group and the others as the non-commitment group. In a comparison of 
the characteristics of the commitment and non-commitment groups, a t-test was employed to compare continuous data, and a chi-square test was used to compare categorical data.

To examine changes in suicidal ideation from the baseline period to the intervention period, we conducted a paired t-test with time of measurement as the independent variable and suicidal ideation as the dependent variable and calculated the effect size Cohen's $d$ in the total sample and each group. To examine the impact of commitment to gratitude intervention on the change in suicidal ideation, a multivariate regression analysis (forced entry method) was used to predict the difference in the mean of suicidal ideation between the baseline and intervention periods. The model includes commitment to gratitude intervention as the independent variable and the following variables as control variables: mean suicidal ideation in the baseline period, sex, age, marital status, family structure, employment, educational background, annual household income, and present psychiatric hospital visit. Regression coefficients $(B)$ and their $95 \%$ confidence intervals $(\mathrm{CI})$ and standardized regression coefficient $(\beta)$ were calculated in the model. The P-values presented are for two-tailed tests. The analysis was performed using SPSS software (SPSS 24.0 for Windows; SPSS Inc., Chicago, IL).

\section{Results}

Table 1 summarizes the participant characteristics. Data for 225 participants were analyzed: $49.3 \%$ were women and the mean age was 40.4 years ( $\mathrm{SD}=11.0)$. Twenty participants $(8.9 \%)$ were committed to the gratitude intervention. The commitment group had a statistically significant higher proportion of people with a high annual household income.

Table 2 summarizes the changes in suicidal ideation from the baseline to the intervention period. Data analysis from all participants and the non-commitment group showed that the mean of suicidal ideation in the baseline period was the same as the mean of suicidal ideation in the intervention period. However, in the commitment group, suicidal ideation was lower in the intervention periods than in the baseline periods. As a result of multiple linear regression analysis, a significant regression equation was found $(F(22,202)=2.86 p<0.001)$, with an $R^{2}$ of 0.24 . Commitment to gratitude intervention was significantly associated with reduced suicidal ideation $(B$ $=-0.67, p=0.015$ ), after controlling for mean suicidal ideation in the baseline period and other demographic variables. 


\section{Discussion}

\section{Principal Findings}

As a result of the implementation of the program on the internet, the dropout rate during the baseline period (about 4 weeks) was 16.5\% ( $n=65)$ and during the intervention period (about 4 weeks) was $24.5 \%(n=81)$ (see Figure 1). The dropout rate is higher than that of previous studies conducted on hospitalized patients (Ducasse et al., 2019; Huffman et al., 2014). In addition, the percentage of those who properly committed to gratitude intervention was very low (8.9\%). Ingenuity is needed to continue receiving interventions on the internet. On the other hand, a small suicide prevention effect $(d=0.22)$ was observed for those who appropriately committed to gratitude intervention. The results of this study are consistent with those of previous studies (Ducasse et al., 2019; Huffman et al., 2014) that have shown the suicide preventive potential of gratitude intervention. Those who are at high risk of suicide do not necessarily visit hospitals, and alternative methods of support are needed. This study demonstrated the feasibility and utility of using the internet to provide gratitude interventions to a larger number of suicidal individuals.

\section{Limitations}

The results of this study should be interpreted in the context of some limitations. First, this was a single-arm study and there was no control group. In the future, rigorous verification of the effects using randomized controlled trials is necessary. Second, this study did not include a follow-up period, so it is unclear how long the effects of the intervention lasted. Third, this study was conducted through an internet survey company. Therefore, the sample used in this study is not necessarily representative of the general population in Japan.

\section{Conclusion \& Future Research}

This study showed that an appropriate commitment to gratitude intervention can lead to a small suicide prevention effect. However, it was difficult to continue implementing this intervention only via the internet. If we can create a way to properly commit to gratitude intervention, it may help reduce suicidal ideation among internet users who do not necessarily visit psychiatric hospitals. In addition, a combination of internet-based and face-to-face interventions may be able to provide more effective support. 


\section{Acknowledgements}

This work was financially supported by JSPS Grants-in-Aid for young scientists Grant Number 18K13358. The funders had no role in study design, data collection and analysis, decision to publish, or preparation of the manuscript.

\section{References}

Beck, A. T., Kovacs, M., \& Weissman, A. (1979). Assessment of suicidal intention: The Scale for Suicide Ideation. Journal of Consulting \& Clinical Psychology, 47(2), 343-352. https://doi.org/10.1037/0022-006X.47.2.343

Ducasse, D., Dassa, D., Courtet, P., Brand-Arpon, V., Walter, A., Guillaume, S., Jaussent, I., \& Olié, E. (2019). Gratitude diary for the management of suicidal inpatients: A randomized controlled trial. Depression \& Anxiety, 36(5), 400-411. https://doi.org/10.1002/da.22877

Emmons, R. A., \& Mccullough, M. E. (2003). Counting blessings versus burdens: An experimental investigation of gratitude and subjective well-being in daily life. Journal of Personality \& Social Psychology, 84(2), 377-389. https://doi.org/10.1037/0022-3514.84.2.377

Huffman, J. C., DuBois, C. M., Healy, B. C., Boehm, J. K., Kashdan, T. B., Celano, C. M., Denninger, J. W., \& Lyubomirsky, S. (2014). Feasibility and utility of positive psychology exercises for suicidal inpatients. General Hospital Psychiatry, 36(1), 88-94. https://doi.org/10.1016/j.genhosppsych.2013.10.006

Japanese Ministry of Internal Affairs and Communications. (2016). 2015 Census. Retrieved from https://www.stat.go.jp/data/kokusei/2015/kekka.html (Accessed: August 20, 2020)

Kleiman, E. M., Adams, L. M., Kashdan, T. B., \& Riskind, J. H. (2013). Grateful individuals are not suicidal: Buffering risks associated with hopelessness and depressive symptoms. Personality \& Individual Differences, 55(5), 595-599. https://doi.org/10.1016/j.paid.2013.05.002

Krysinska, K., Lester, D., Lyke, J., \& Corveleyn, J. (2015). Trait gratitude and suicidal ideation and behavior. Crisis, 36(4), 291-296. https://doi.org/10.1027/0227-5910/a000320

Sueki, H. (2019). Development of a short form of the suicidal ideation scale. Suicide Prevention \& Crisis Intervention, 39(2), 94-101. (Japanese) 


\section{Figure 1}

\section{Sampling process}

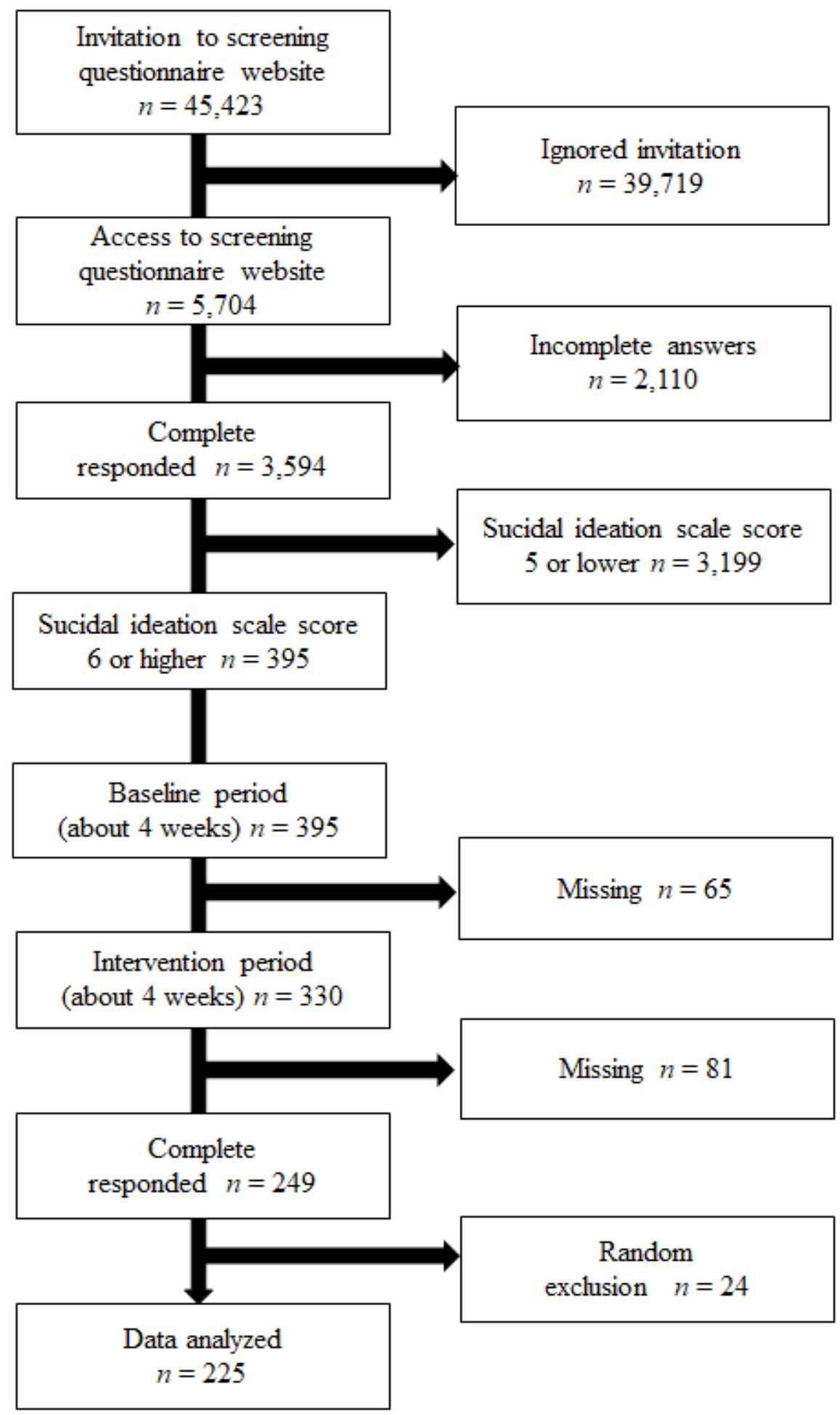


Table 1

Characteristics of study participants

\begin{tabular}{|c|c|c|c|c|c|c|c|}
\hline & \multicolumn{2}{|c|}{ Total } & \multicolumn{2}{|c|}{ Commitment group } & \multicolumn{2}{|c|}{ Non-commitment group } & \multirow{2}{*}{$p$} \\
\hline & $n /$ mean & $\% / \mathrm{SD}$ & $n /$ mean & $\% / \mathrm{SD}$ & $n /$ mean & $\% / \mathrm{SD}$ & \\
\hline Sex; woman & 111 & 49.3 & 10 & 50.0 & 101 & 49.3 & 0.950 \\
\hline Age; mean, SD & 40.4 & 11.0 & 40.7 & 11.6 & 40.4 & 10.8 & 0.898 \\
\hline 20 's & 47 & 20.9 & 5 & 25.0 & 42 & 20.5 & \\
\hline 30 's & 66 & 29.3 & 4 & 20.0 & 62 & 30.2 & \\
\hline 40 's & 55 & 24.4 & 3 & 15.0 & 52 & 25.4 & 0.338 \\
\hline 50 's & 53 & 23.6 & 8 & 40.0 & 45 & 22.0 & \\
\hline over 60's & 4 & 1.8 & 0 & 0.0 & 4 & 2.0 & \\
\hline \multicolumn{8}{|l|}{ Marital status } \\
\hline married & 63 & 28.0 & 7 & 35.0 & 56 & 27.3 & \multirow{4}{*}{0.856} \\
\hline bereaved & 1 & 0.4 & 0 & 0.0 & 1 & 0.5 & \\
\hline divorced & 18 & 8.0 & 1 & 5.0 & 17 & 8.3 & \\
\hline not married & 143 & 63.6 & 12 & 60.0 & 131 & 63.9 & \\
\hline Without children & 172 & 76.4 & 16 & 80.0 & 156 & 76.1 & 0.695 \\
\hline \multicolumn{8}{|l|}{ Employment } \\
\hline permanent employee & 83 & 36.9 & 8 & 40.0 & 75 & 36.6 & \multirow{6}{*}{0.780} \\
\hline part-time, temporary worker & 49 & 21.8 & 4 & 20.0 & 45 & 22.0 & \\
\hline self-employed & 13 & 5.8 & 0 & 0.0 & 13 & 6.3 & \\
\hline unemployed & 43 & 19.1 & 4 & 20.0 & 39 & 19.0 & \\
\hline full-time homemaker & 20 & 8.9 & 3 & 15.0 & 17 & 8.3 & \\
\hline other & 17 & 7.6 & 1 & 5.0 & 16 & 7.8 & \\
\hline \multicolumn{8}{|l|}{ Educational background } \\
\hline junior high school / high school & 80 & 35.6 & 8 & 40.0 & 72 & 35.1 & \multirow{3}{*}{0.768} \\
\hline specialized training college / two-year college & 49 & 21.8 & 5 & 25.0 & 44 & 21.5 & \\
\hline university / graduate school & 96 & 42.7 & 7 & 35.0 & 89 & 43.4 & \\
\hline \multicolumn{8}{|l|}{ Household income } \\
\hline unknown & 47 & 20.9 & 5 & 25.0 & 42 & 20.5 & \multirow{4}{*}{0.037} \\
\hline less than JPY 400 million & 78 & 34.7 & 3 & 15.0 & 75 & 36.6 & \\
\hline JPY 400-800 million & 66 & 29.3 & 5 & 25.0 & 61 & 29.8 & \\
\hline more than JPY 800 million & 34 & 15.1 & 7 & 35.0 & 27 & 13.2 & \\
\hline Present psychiatric hospital visit & 71 & 31.6 & 7 & 35.0 & 64 & 31.2 & 0.728 \\
\hline
\end{tabular}


Table 2

Changes in suicidal ideation from the baseline period to the intervention period

\begin{tabular}{lccccccccc}
\hline & \multicolumn{7}{c}{ Baseline } & \multicolumn{2}{c}{ Intervention } \\
& & Mean & SD & Mean & SD & $d$ & $t$ & $p$ \\
\hline Total & 225 & 7.19 & 2.67 & 7.22 & 3.13 & 0.01 & 0.42 & 0.678 \\
Commitment Group & 20 & 6.25 & 2.54 & 5.59 & 3.42 & 0.22 & 2.05 & 0.055 \\
Non-Commitment Group & 205 & 7.28 & 2.67 & 7.38 & 3.06 & 0.04 & 1.24 & 0.217 \\
\hline
\end{tabular}




\section{Appendix 1}

The 6-item Suicidal Ideation Scale

1. Do you wish to die? (0. None, 1 . Weak, 2. Moderate to strong)

2. Please describe the balance between your desire to live and your desire to die. ( 0 . The desire to live is stronger than the desire to die, 1 . Both are about the same, 2. The desire to die is stronger than the desire to live.)

3. When you have suicidal thoughts, do you have the desire to actively attempt suicide? (0. None, 1. Weak, 2. Moderate to strong)

4. When you have suicidal thoughts, how long do they last? (0. Brief, fleeting periods: 1. Longer periods, 2. Continuous (chronic)

5. How often do you have suicidal thoughts? (0. Rare, occasional, 1. Intermittent, 2. Persistent or Continuous

6. Have you made plans for a suicide attempt? (0. Not considered, 1 . Considered, but details not worked out, 2. Details worked out/well formulated.) 